\title{
ARRANJOS DE MORADIA DE IDOSOS: ASSOCIAÇÃO COM INDICADORES SOCIODEMOGRÁFICOS E DE SAÚDE
}

Elderly Housing Arrangements: Association with Sociodemographic and Health Indicators

Arreglos de Vivienda de Ancianos: Asociación con Indicadores Socio Demográficos y de Salud

Logement des Personnes Agées : Association avec Indicateurs Sociodémographiques et Sanitaires

10.5020/23590777.rs.v20iEsp1.e8873

\begin{abstract}
Dóris Firmino Rabelo (9)
Doutora em Educação pela Universidade Estadual de Campinas (UNICAMP). Atualmente é professora da Universidade Federal do Recôncavo da Bahia (UFRB) e do Programa de Pós-Graduação em Psicologia da Universidade Federal da Bahia (UFBA).
\end{abstract}

\section{Nara Maria Forte Diogo Rocha 9}

Doutora em Educação Brasileira pela Universidade Federal do Ceará (UFC). Docente da Universidade Federal do Ceará (UFC).

\section{Juliana Martins Pinto 9}

Pós-Doutorado em Fisioterapia e Epidemiologia do Envelhecimento na Universidade Federal do Rio Grande do Norte (UFRN). Atualmente é professora na Universidade Federal do Triângulo Mineiro (UFTM).

\section{Resumo}

O objetivo deste estudo foi analisar o arranjo de moradia, como um indicador de vulnerabilidade social, e suas relações com indicadores sociodemográficos e de saúde de idosos do interior da Bahia. Trata-se de um estudo transversal com amostra de 134 idosos, entrevistados em domicílio. Avaliou-se os arranjos de moradia e suas associações com sexo; idade; chefia familiar; contribuição financeira; número de doenças, sinais e sintomas; desempenho em atividades de vida diária e envolvimento social. Foram realizados testes estatísticos para comparação de frequência entre os grupos (qui-quadrado e exato de Fisher). Viver sozinho ou acompanhado, com ou sem descendentes, teve associações com indicadores sociodemográficos (chefia e contribuição financeira). Viver com ou sem cônjuge associou-se com medidas de saúde, sexo e idade, o que pode indicar a importância desse membro familiar para o bem-estar do idoso.

Palavras-chave: família; vulnerabilidade social; saúde do idoso; participação social; envelhecimento.

\section{Abstract}

The aim of this study was to analyze the housing arrangement, as an indicator of social vulnerability, and its relationship with sociodemographic and health indicators of the elderly in the countryside of Bahia. This is a cross-sectional study with a sample of 134 elderly people interviewed at home. Housing arrangements and their associations with sex; age; family head; financial contribution; the number of diseases, signs, and symptoms; performance in activities of daily living, and social involvement were evaluated. Statistical tests were performed to compare the frequency between groups (chi-square and Fisher's exact). Living alone or accompanied, with or without descendants, comprise associations with sociodemographic indicators (leadership and financial contribution). Living with or without a spouse was associated with health, sex and age measures, which may indicate the importance of this family member for the well-being of the elderly.

Keywords: family; social vulnerability; elderly health; social participation; aging. 


\section{Resumen}

El objetivo de este trabajo fue analizar el arreglo de vivienda, como indicador de vulnerabilidad social y sus relaciones con indicadores socio demográficos y de salud de ancianos en el interior de Bahia. Este estudio transversal cuenta con una muestra de 134 ancianos, entrevistados en viviendas. Fueron evaluados los arreglos de vivienda y sus asociaciones con sexo; edad; cabeza de familia; contribución financiera; número de enfermedades, señales y síntomas; rendimiento en actividades de la vida diaria y participación social. Fueron realizados análisis estadísticos para comparación de frecuencia entre los grupos (chi-cuadrado y exacto de Fisher). Vivir solo o acompañado, con o sin descendientes, tuvo asociaciones con indicadores socio demográfico (dirección y contribución financiera). Vivir con o sin cónyuge se relacionó con medidas de salud, sexo y edad, lo que puede indicar la importancia de este miembro familiar para el bienestar del anciano.

Palabras clave: familia; vulnerabilidad social; salud del anciano; participación social; envejecimiento.

\section{Résumé}

Le but de cette étude a été d'analyser l'agencement du logement, en tant qu'indicateur de vulnérabilité sociale. En autre, on a aussi étudié sa relation avec les indicateurs sociodémographiques et sanitaires des personnes âgées à l'intérieur de l'état de la Bahia, au Brésil. Il s'agit d'une étude transversale avec un échantillon de 134 personnes âgées interrogées à domicile. Les modalités de logement et leurs associations avec le sexe, l'âge; le chef de famille ; la contribution financière; le nombre de maladies, les symptômes; la performance dans les activités de la vie quotidienne et l'engagement social. Des tests statistiques ont été effectués pour comparer la fréquence entre les groupes (chi carré et exact de Fisher). Isolé ou accompagné, avec ou sans descendance, ont été associés à des indicateurs sociodémographiques (chef de famille et contribution financière). Vivre avec ou sans épouse a été associé à des mesures sanitaire, de sexe et d'âge, ce qui peut indiquer l'importance de ce membre de la famille pour le bien-être des personnes âgées.

Mots-clés : famille ; vulnérabilité sociale ; santé des personnes âgées ; participation sociale ; vieillissement.

A compreensão do envelhecer como um processo heterogêneo e profundamente afetado pelas condições sociais tem exigido uma maior atenção a fatores implicados na vulnerabilidade social de idosos brasileiros, entre as quais os arranjos de moradia são centrais. O arranjo de moradia, em suas diversas configurações, é ressaltado pelos idosos como um aspecto da sua qualidade de vida (Carmo \& Guizardi, 2018; Fontes \& Neri, 2015).

Os tipos de arranjo de moradia em que os idosos estão inseridos correspondem a um importante indicador da vulnerabilidade social a qual estão expostos (Andrew, Mitnitski, \& Rockwood, 2008; Campos, Rezende, Ferreira, Vargas, \& Gonçalves, 2017; Pavarini et al., 2009; Perseguino, Horta, \& Ribeiro, 2017). Com o aumento da população idosa, as características dos arranjos de moradia e a constituição familiar têm sofrido importantes mudanças, tanto em sua estrutura como na dinâmica das relações que compõem esses arranjos (Ferreira, 2017). Essas mudanças influenciam de maneira expressiva a maneira como as pessoas envelhecem e a prestação de cuidados e serviços de saúde aos idosos no âmbito familiar (Del Duca, Martinez, \& Bastos, 2012; Faleiros et al., 2015; Giacomin, Duarte, Camarano, Nunes, \& Fernandes, 2018).

A família ainda é central no tocante à moradia dos idosos, sendo uma escolha ou uma imposição social. Por outro lado, morar sozinho tem se consolidado como tendência devido à mudança das configurações familiares relativas à quantidade de filhos e recomposições. Esse tipo de arranjo pode tanto pressupor um envelhecimento saudável, devido às características de independência e autonomia necessárias, como pode revelar-se um fator de fragilidade para a saúde física e mental de idosos (Freire \& Junior, 2017).

A moradia constitui-se como importante para o suporte da identidade. Considerada lugar de convivência familiar e comunitária idealmente propiciadora de cuidados, revela os arranjos e reorganizações (coletivas e individuais) que foram exigidas ao longo da vida, o que destaca seu potencial nas análises relativas à vulnerabilidade social dessa população. Uma visão ampla da moradia para os idosos permite analisar suas articulações com a saúde e a assistência, dando maiores subsídios para a compreensão da vulnerabilidade social. Além disso, os arranjos de moradia impactam na saúde física e psicológica, bem como se relacionam com os papéis sociais desempenhados pelos idosos no domicílio, de modo que a constituição do arranjo em que o idoso está inserido tem implicações fundamentais para o seu envelhecimento (Freire \& Junior, 2017).

$\mathrm{Na}$ sociedade brasileira, a coabitação tem um forte papel na integração dos membros que compõem o domicílio, sendo que, em geral, a distribuição de direitos e obrigações é mais intensa entre as pessoas que vivem em uma mesma residência. $\mathrm{Na}$ velhice, as relações estabelecidas com os membros do arranjo de moradia, familiares ou não, implicam em maior ou menor suporte social, em oferta de cuidados, bem como em condições financeiras e de saúde (Fontes, Fortes-Burgos, Mello, Pereira, \& Neri, 2011; Freire \& Junior, 2017; Kooshiar, Yahaya, Hamid, Samah, \& Jou, 2012). 
Na velhice, a saúde é reconhecida como o principal alvo de preocupações e expectativas, tanto para os idosos e cuidadores familiares como para as pessoas que trabalham com essa população. Diversos estudos apontaram a importância dos arranjos de moradia para a saúde (Campos et al., 2017; Russell \& Taylor, 2009; Salmazo-Silva et al., 2012), para a funcionalidade (Campos et al., 2017; Russell \& Taylor, 2009; Wang, Chen, Pan, Jing, \& Liu, 2013), para o acesso aos serviços de saúde (Gomes, Turra, Fígoli, Duarte, \& Lebrão, 2013; Kasper, Pezzin, \& Rice, 2010; Lau \& Kirby, 2009), para a longevidade (Lima-Costa, Matos, Camargos, \& Macinko, 2011; Manfredini \& Breschi, 2013) e para o bem-estar e qualidade de vida dos idosos (Campos et al., 2017; Kooshiar et al., 2012; Neri et al., 2018).

Desse modo, conhecer os diferentes arranjos de moradia nos quais os idosos vivem e compreender as relações destes com indicadores sociodemográficos e de saúde é fundamental para entender a conjuntura sob a qual se envelhece, contribuindo para aprimorar a abordagem do idoso em diversos contextos sociais. Assim, o objetivo deste estudo foi analisar o arranjo de moradia de idosos, como um indicador de vulnerabilidade social, e suas relações com variáveis sociodemográficas (faixa etária, sexo, chefia familiar, contribuição financeira familiar) e de saúde (número de doenças, sinais e sintomas autorrelatados, desempenho em atividades básicas e instrumentais de vida diária, e envolvimento social).

\section{Método}

\section{Contexto do Estudo}

A pesquisa foi realizada no chamado Território de Identidade Recôncavo, localizado na região leste do estado da Bahia, com IDH calculado em 0,621 e índice de GINI de 0,574, o que indica um contexto de grandes desigualdades sociais (SEI, 2016). Os(as) idosos(as) residiam em Santo Antônio de Jesus, cidade polo da região, um importante centro comercial e de serviços, regionalmente considerada como a capital do Recôncavo.

\section{Participantes e Procedimentos}

Participaram do estudo 134 idosos(as), com idade entre 60 e 95 anos, a maioria do sexo feminino (77,6\%). A coleta dos dados foi realizada no ano de 2011, na Unidade Básica de Saúde (UBS) que tinha o maior número de idosos cadastrados (16,8\% dos idosos residentes na cidade) na época da coleta de dados. Segundo os dados do Sistema de Informação da Atenção Básica - SIAB (2010), estavam cadastradas 2.754 famílias, que somavam 9.234 pessoas, sendo 1344 idosos, o que representa $14,5 \%$ dos cadastros nessa UBS.

Com a ajuda de agentes comunitários de saúde, foi realizada a identificação dos domicílios com idosos localizados em todas as microáreas abrangidas pela UBS selecionada. Os idosos foram abordados no domicílio e convidados a participar da pesquisa. Os recrutadores foram instruídos para considerar os seguintes critérios de elegibilidade: idade igual ou superior a 60 anos; residência permanente na região e no domicílio; compreensão das instruções; interesse em participar; e assinatura do Termo de Consentimento Livre e Esclarecido. O critério de inclusão na amostra baseou-se no desempenho dos idosos no Mini-Exame do Estado Mental (MEEM), um teste de rastreio de demências comumente usado em estudos populacionais. Foram adotadas notas de corte segundo os padrões adotados no estudo FIBRA (Fontes et al., 2011; Pinto \& Neri, 2013). A opção pela inclusão de idosos sem déficit cognitivo sugestivo de demência foi feita para não prejudicar a confiabilidade das respostas de autorrelato dos idosos. Foram visitados 207 domicílios com idosos, dos quais 7 (3,4\%) não cumpriam um ou mais critérios de elegibilidade. Sessenta e seis idosos (31,9\%) pontuaram abaixo da nota de corte no MEEM, razão pela qual foram excluídos.

Foi solicitado ao idoso que a entrevista ocorresse em um local da casa que pudesse proporcionar maior privacidade $\mathrm{e}$ tranquilidade, evitando-se a interrupção por outras pessoas. A coleta durou de duas a três visitas, de 2 horas cada uma, em média. Todos os idosos assinaram um termo de consentimento e o estudo foi devidamente aprovado por um comitê de ética em pesquisa, seguindo as especificações da Resolução n. ${ }^{\circ}$ 466, de 12 de dezembro, do Conselho Nacional de Saúde. (2012).

\section{Variáveis e Instrumentos}

\section{Questionário de informações sociodemográficas.}

Foram registradas: a) idade, agrupada em duas faixas (60 a 74 anos e 75 anos ou mais); b) sexo (feminino/masculino); c) chefia familiar, avaliada com base na pergunta: “quem é o chefe da família?” As respostas foram agrupadas nas opções sim (o idoso era chefe da família) ou não (o idoso não era o chefe da família), de modo que foi registrado se o idoso entrevistado era ou não o chefe da família; d) contribuição financeira do idoso para o sustento da família (uma pergunta com as alternativas total, parcial ou nenhuma); e) arranjos de moradia, com base na pergunta: "com quem o(a) Sr(a) mora?" As opções foram: 
sozinho(a), com o(a) cônjuge ou companheiro(a), com filhos(as), netos(as), outros parentes e pessoas de fora da família. As respostas foram agrupadas nas categorias: sozinho, com o cônjuge ou companheiro, com o cônjuge e descendentes, com descendentes e outros tipos de arranjo.

\section{Indicadores de saúde.}

Questionário de doenças e de sinais e sintomas autorrelatados (Fontes et al., 2011).

Perguntou-se aos idosos sobre as doenças crônicas que foram diagnosticadas pelo médico no último ano (doenças cardíacas, hipertensão, derrame, diabetes, câncer, artrite/reumatismo, bronquite/enfisema, depressão e osteoporose) e, para cada uma das doenças, o idoso respondia sim ou não. O mesmo procedimento foi adotado para avaliar sinais e sintomas (dor, inchaço, falta de ar, vertigem, fadiga, tosse, transpiração excessiva ou sede persistente, quedas, ganho ou perda de peso involuntário, dificuldade de memória). Ambas as variáveis foram categorizadas em: nenhuma; 1 a 2; 3 ou mais.

\section{Independência nas atividades básicas da vida diária (ABVDs) (Katz, Ford, Moskowitz, Jackson, \& Jaffe, 1963).}

Foi avaliada mediante escala com três possibilidades de resposta sobre a ajuda necessária (nenhuma, parcial ou total) para banhar-se, vestir-se, alimentar-se, usar o banheiro, andar e transferir-se, e manter-se continente. As respostas foram agrupadas em: independente, dependência parcial e dependência total.

\section{Desempenho em atividades instrumentais de vida diária (AIVDs) (Lawton \& Brody, 1969).}

Foi avaliado mediante escala com três possibilidades de resposta sobre a ajuda necessária (nenhuma, parcial ou total) para telefonar, usar transportes, comprar, cozinhar, realizar serviços domésticos, usar medicação e manejar dinheiro. As respostas foram agrupadas em: independente, dependência parcial e dependência total.

\section{Envolvimento social.}

O envolvimento social foi baseado em autorrelato sobre a participação em atividades físicas, religiosas, em centros de convivência e na comunidade (quatro questões com respostas que foram agrupadas em sim ou não). Os dados foram categorizados pela mediana, em menor ou maior envolvimento social.

\section{Análise de Dados}

Os dados foram submetidos à análise estatística com a utilização do programa Statistical Package for Social Sciences ${ }^{\circledR}$ (SPSS), versão 19.0. Foram utilizados o teste qui-quadrado e o teste exato de Fisher (na presença de valores esperados menores que 5) para comparar as variáveis de interesse com os grupos de arranjo de moradia. Para comparar os grupos de diferentes arranjos de moradia segundo a vulnerabilidade social a qual os idosos estavam sujeitos, dividiram-se os arranjos em três grupos, sendo eles: sozinho X acompanhado (com cônjuge, descendentes ou outros); com cônjuge X sem cônjuge (sozinho, com descendentes e outros) e com descendentes X sem descendentes (sozinho, com cônjuge e outros). Para cada grupo foram comparadas as frequências e as associações com os indicadores sociodemográficos e de saúde. O nível de significância adotado para os testes estatísticos foi de $5 \%$, ou seja, $\mathrm{p}<0,05$.

\section{Resultados}

A idade dos participantes variou entre 60 a 95 anos, sendo $62,7 \%$ na faixa etária entre 60 e 74 anos e $37,5 \%$ com 75 anos ou mais; a maioria do sexo feminino; chefe de família; que contribuíam totalmente ou parcialmente para o sustento da família. A maior parte vivia com descendentes, seguidos por aqueles que viviam em arranjos mistos (cônjuge e descendentes), pelos que viviam sozinhos e pelos que viviam em outros tipos de arranjo ou só com o cônjuge (Tabela 1).

Quanto às condições de saúde, a maioria relatou ter uma ou duas doenças, três ou mais sinais e sintomas, pelo menos uma doença crônica e pelo menos um sinal ou sintoma. Por outro lado, a maior parte deles se percebia independente, tanto para as atividades básicas como para as instrumentais de vida diária. A maioria dos idosos mantinham níveis baixos de envolvimento em atividades sociais (Tabela 1). 
Tabela 1

Caracterização da amostra segundo os indicadores sociodemográficos e de saúde e os arranjos de moradia

\begin{tabular}{|c|c|c|c|}
\hline Variáveis & Categorias & $f$ & $\%$ \\
\hline \multirow{2}{*}{ Faixa etária } & $60-74$ & 84 & 62,7 \\
\hline & $75+$ & 50 & 37,3 \\
\hline \multirow{2}{*}{ Sexo } & Feminino & 104 & 77,6 \\
\hline & Masculino & 30 & 22,4 \\
\hline \multirow{2}{*}{ Chefia familiar } & Sim & 97 & 72,4 \\
\hline & Não & 37 & 27,6 \\
\hline \multirow{3}{*}{ Contribuição financeira familiar } & Total & 66 & 49,3 \\
\hline & Parcial & 59 & 44 \\
\hline & Nenhuma & 9 & 6,7 \\
\hline \multirow{3}{*}{$\mathrm{N}^{\mathrm{o}}$ de doenças } & 0 & 18 & 13,4 \\
\hline & $1-2$ & 70 & 52,2 \\
\hline & $3+$ & 46 & 34,3 \\
\hline \multirow{3}{*}{$\mathrm{N}^{\mathrm{o}}$ de sinais e sintomas } & 0 & 12 & 9 \\
\hline & $1-2$ & 42 & 31,3 \\
\hline & $3+$ & 80 & 59,7 \\
\hline \multirow{3}{*}{ ABVDs } & Independente & 129 & 96,3 \\
\hline & Dependência parcial & 3 & 2,2 \\
\hline & Dependência total & 2 & 1,5 \\
\hline \multirow{3}{*}{ AIVDs } & Dependência total & 8 & 6 \\
\hline & Dependência parcial & 48 & 35,8 \\
\hline & Independente & 78 & 58,2 \\
\hline \multirow{2}{*}{ Envolvimento social } & Baixo & 88 & 65,7 \\
\hline & Alto & 46 & 34,3 \\
\hline \multirow{5}{*}{ Arranjos de moradia } & Sozinho & 24 & 17,9 \\
\hline & Com cônjuge & 10 & 7,5 \\
\hline & Com descendentes & 55 & 41 \\
\hline & Misto & 32 & 23,9 \\
\hline & Outros & 13 & 9,7 \\
\hline
\end{tabular}

Nota. ABVDs: atividades básicas de vida diária; AIVDs: atividades instrumentais de vida diária.

A análise comparativa das variáveis mostrou que houve diferença significativa entre os grupos de arranjo de moradia de acordo com: sexo (maior frequência de sexo masculino entre os idosos que moram somente com o cônjuge e os que moram com o cônjuge e seus descendentes); idade (maior frequência de idosos com idade $\geq 75$ anos entre os que moram com o cônjuge); chefia familiar (maior frequência de chefia familiar entre aqueles idosos que moram sozinhos, em arranjos mistos com outros e com os descendentes); contribuição financeira (maior frequência de contribuição total para o sustento da família entre os que moram sozinhos); envolvimento social (os idosos que vivem em arranjos mistos foram mais envolvidos quando comparados com os demais grupos) (Tabela 2).

Tabela 2

Comparação entre os indicadores sociodemográficos e de saúde segundo os arranjos de moradia

\begin{tabular}{|c|c|c|c|c|c|c|c|}
\hline \multirow{2}{*}{ Variáveis } & \multirow{2}{*}{ Categorias } & \multicolumn{5}{|c|}{ Arranjos de moradia } & \multirow{2}{*}{$p$} \\
\hline & & Sozinho & Cônjuge & Desc & Misto & Outros & \\
\hline \multirow{2}{*}{ Faixa etária } & $60-74$ & 13 & 3 & 30 & 28 & 10 & \multirow{2}{*}{$0,003 *$} \\
\hline & $75+$ & 11 & 7 & 25 & 4 & 3 & \\
\hline \multirow{2}{*}{ Sexo } & Feminino & 18 & 5 & 53 & 18 & 10 & \multirow{2}{*}{$<0,001^{*}$} \\
\hline & Masculino & 6 & 5 & 2 & 14 & 3 & \\
\hline \multirow{2}{*}{ Chefia familiar } & Sim & 24 & 5 & 42 & 16 & 10 & \multirow{2}{*}{$<0,001$} \\
\hline & Não & 0 & 5 & 13 & 16 & 3 & \\
\hline
\end{tabular}




\begin{tabular}{|c|c|c|c|c|c|c|c|}
\hline \multirow{3}{*}{ Contribuição financeira familiar } & Total & 23 & 3 & 26 & 8 & 6 & \multirow{3}{*}{$<0,001 \nRightarrow$} \\
\hline & Parcial & 1 & 5 & 26 & 20 & 7 & \\
\hline & Nenhuma & 0 & 2 & 3 & 4 & 0 & \\
\hline \multirow{3}{*}{$\mathrm{N}^{\mathrm{o}}$ de doenças } & 0 & 2 & 4 & 7 & 4 & 1 & \multirow{3}{*}{0,278} \\
\hline & $1-2$ & 11 & 3 & 32 & 18 & 6 & \\
\hline & $3+$ & 11 & 3 & 10 & 6 & 16 & \\
\hline \multirow{3}{*}{$\mathrm{N}^{\mathrm{o}}$ de sinais e sintomas } & 0 & 2 & 1 & 4 & 3 & 2 & \multirow{3}{*}{0,972} \\
\hline & $1-2$ & 6 & 3 & 18 & 12 & 3 & \\
\hline & $3+$ & 16 & 6 & 33 & 17 & 8 & \\
\hline \multirow{3}{*}{ ABVDs } & Independente & 22 & 10 & 53 & 31 & 13 & \multirow{3}{*}{0,749} \\
\hline & Dependência parcial & 1 & 0 & 2 & 0 & 0 & \\
\hline & Dependência total & 1 & 0 & 0 & 1 & 0 & \\
\hline \multirow{3}{*}{ AIVDs } & Dependência total & 3 & 1 & 3 & 1 & 0 & \multirow{3}{*}{0,144} \\
\hline & Dependência parcial & 8 & 2 & 27 & 7 & 4 & \\
\hline & Independente & 13 & 7 & 25 & 24 & 9 & \\
\hline \multirow{2}{*}{ Envolvimento social } & Baixo & 15 & 5 & 43 & 15 & 10 & \multirow{2}{*}{$0,029^{*}$} \\
\hline & Alto & 9 & 5 & 12 & 17 & 3 & \\
\hline
\end{tabular}

Nota. ABVDs: atividades básicas de vida diária; AIVDs: atividades instrumentais de vida diária; Desc: descendentes; $p$ significância estatística $(<0,05)$;

* Teste qui-quadrado; \# Teste exato de Fisher.

Observaram-se associações entre os arranjos de moradia (sozinho $\mathrm{x}$ acompanhado) com chefia familiar e contribuição financeira do idoso para com a família, sendo que os idosos que viviam sozinhos foram mais frequentes entre aqueles que chefiavam a família, bem como entre aqueles que contribuíam totalmente para o sustento da família (Tabela 3).

Tabela 3

Comparação entre os indicadores sociodemográficos e de saúde segundo os arranjos de moradia sozinho e acompanhado

\begin{tabular}{|c|c|c|c|c|}
\hline \multirow{2}{*}{ Variáveis } & \multirow{2}{*}{ Categorias } & \multicolumn{2}{|c|}{ Arranjos de moradia } & \multirow{2}{*}{$p$} \\
\hline & & Sozinho & Acompanhado & \\
\hline \multirow{2}{*}{ Faixa etária } & $60-74$ & 13 & 71 & \multirow{2}{*}{0,234} \\
\hline & $75+$ & 11 & 39 & \\
\hline \multirow{2}{*}{ Sexo } & Feminino & 18 & 86 & \multirow{2}{*}{0,460} \\
\hline & Masculino & 6 & 24 & \\
\hline \multirow{2}{*}{ Chefia familiar } & Sim & 24 & 73 & \multirow{2}{*}{$<0,001 \#$} \\
\hline & Não & 0 & 37 & \\
\hline \multirow{3}{*}{$\begin{array}{l}\text { Contribuição financeira } \\
\text { familiar }\end{array}$} & Total & 23 & 43 & \multirow{3}{*}{$<0,001^{*}$} \\
\hline & Parcial & 1 & 58 & \\
\hline & Nenhuma & 0 & 9 & \\
\hline \multirow{3}{*}{$\mathrm{N}^{\circ}$ de doenças } & 0 & 2 & 16 & \multirow{3}{*}{0,382} \\
\hline & $1-2$ & 11 & 59 & \\
\hline & $3+$ & 11 & 35 & \\
\hline \multirow{3}{*}{$\mathrm{N}^{\mathrm{o}}$ de sinais e sintomas } & 0 & 2 & 10 & \multirow{3}{*}{0,731} \\
\hline & $1-2$ & 6 & 36 & \\
\hline & $3+$ & 16 & 64 & \\
\hline \multirow{3}{*}{ ABVDs } & Independente & 22 & 107 & \multirow{3}{*}{0,377} \\
\hline & Dependência parcial & 1 & 2 & \\
\hline & Dependência total & 1 & 1 & \\
\hline \multirow{3}{*}{ AIVDs } & Dependência total & 3 & 5 & \multirow{3}{*}{0,329} \\
\hline & Dependência parcial & 8 & 40 & \\
\hline & Independente & 13 & 65 & \\
\hline \multirow{2}{*}{ Envolvimento social } & Baixo & 15 & 73 & \multirow{2}{*}{0,444} \\
\hline & Alto & 9 & 37 & \\
\hline
\end{tabular}

Nota. ABVDs: atividades básicas de vida diária; AIVDs: atividades instrumentais de vida diária; $p$ significância estatística $(<0,05)$; * Teste qui-quadrado; \# Teste exato de Fisher. 
$\mathrm{Na}$ comparação entre os arranjos com e sem o cônjuge, observaram-se associações significativas com idade, sexo e número de doenças, sendo os mais velhos (75 anos ou mais) mais frequentes entre aqueles que viviam com o cônjuge e as mulheres mais frequentes entre os idosos que viviam sem o cônjuge. Além disso, os idosos que relataram mais doenças foram mais frequentes entre os idosos que viviam sem o cônjuge (Tabela 4).

Tabela 4

Comparação entre os indicadores sociodemográficos e de saúde segundo os arranjos com cônjuge e sem cônjuge

\begin{tabular}{|c|c|c|c|c|}
\hline \multirow{2}{*}{ Variáveis } & \multirow{2}{*}{ Categorias } & \multicolumn{2}{|c|}{ Arranjos de moradia } & \multirow[b]{2}{*}{$p$} \\
\hline & & Com cônjuge & Sem cônjuge & \\
\hline \multirow{2}{*}{ Faixa etária } & $60-74$ & 3 & 81 & \multirow{2}{*}{$0,032 \#$} \\
\hline & $75+$ & 7 & 43 & \\
\hline \multirow{2}{*}{ Sexo } & Feminino & 5 & 99 & \multirow{2}{*}{$0,045 \#$} \\
\hline & Masculino & 5 & 25 & \\
\hline \multirow{2}{*}{ Chefia familiar } & Sim & 5 & 92 & \multirow{2}{*}{0,104} \\
\hline & Não & 5 & 32 & \\
\hline \multirow{3}{*}{$\begin{array}{l}\text { Contribuição financeira } \\
\text { familiar }\end{array}$} & Total & 3 & 63 & \multirow{3}{*}{0,154} \\
\hline & Parcial & 5 & 54 & \\
\hline & Nenhuma & 2 & 7 & \\
\hline \multirow{3}{*}{$\mathrm{N}^{\mathrm{o}}$ de doenças } & 0 & 4 & 14 & \multirow{3}{*}{$0,034^{*}$} \\
\hline & $1-2$ & 3 & 67 & \\
\hline & $3+$ & 3 & 43 & \\
\hline \multirow{3}{*}{$\mathrm{N}^{\mathrm{o}}$ de sinais e sintomas } & 0 & 1 & 11 & \multirow{3}{*}{0,990} \\
\hline & $1-2$ & 3 & 39 & \\
\hline & $3+$ & 6 & 74 & \\
\hline \multirow{3}{*}{ ABVDs } & Independente & 10 & 119 & \multirow{3}{*}{0,811} \\
\hline & Dependência parcial & 0 & 3 & \\
\hline & Dependência total & 0 & 2 & \\
\hline \multirow{3}{*}{ AIVDs } & Dependência total & 1 & 7 & \multirow{3}{*}{0,520} \\
\hline & Dependência parcial & 2 & 46 & \\
\hline & Independente & 7 & 71 & \\
\hline \multirow{2}{*}{ Envolvimento social } & Baixo & 5 & 83 & \multirow{2}{*}{0,226} \\
\hline & Alto & 5 & 41 & \\
\hline
\end{tabular}

Nota. ABVDs: atividades básicas de vida diária; AIVDs: atividades instrumentais de vida diária; p: significância estatística $(<0,05) ;$ * Teste qui-quadrado; \# Teste exato de Fisher.

Na comparação entre os arranjos com e sem descendentes, foram observadas associações com chefia familiar e contribuição financeira. Os idosos chefes de família e que contribuíam parcial ou totalmente com a renda familiar foram mais frequentes entre aqueles que viviam com os descendentes quando comparados com os idosos que viviam sem os descendentes (Tabela 5).

Tabela 5

Comparação entre os indicadores sociodemográficos e de saúde segundo os arranjos de moradia com e sem descendentes

\begin{tabular}{|c|c|c|c|c|}
\hline \multirow{2}{*}{ Variáveis } & \multirow{2}{*}{ Categorias } & \multicolumn{2}{|c|}{ Arranjos de moradia } & \multirow{2}{*}{$p$} \\
\hline & & Com descendentes & Sem descendentes & \\
\hline \multirow{2}{*}{ Faixa etária } & $60-74$ & 58 & 26 & \multirow{2}{*}{0,134} \\
\hline & $75+$ & 29 & 21 & \\
\hline \multirow{2}{*}{ Sexo } & Feminino & 71 & 33 & \multirow{2}{*}{0,099} \\
\hline & Masculino & 16 & 14 & \\
\hline \multirow{2}{*}{ Chefia familiar } & Sim & 58 & 39 & \multirow{2}{*}{$0,033 \#$} \\
\hline & Não & 29 & 8 & \\
\hline \multirow{3}{*}{ Contribuição financeira familiar } & Total & 34 & 32 & \multirow{3}{*}{$0,006^{*}$} \\
\hline & Parcial & 46 & 13 & \\
\hline & Nenhuma & 7 & 2 & \\
\hline
\end{tabular}




\begin{tabular}{|c|c|c|c|c|}
\hline \multirow{3}{*}{$\mathrm{N}^{\mathrm{o}}$ de doenças } & 0 & 11 & 7 & \multirow{3}{*}{0,242} \\
\hline & $1-2$ & 50 & 20 & \\
\hline & $3+$ & 26 & 20 & \\
\hline \multirow{3}{*}{$\mathrm{N}^{\mathrm{o}}$ de sinais e sintomas } & 0 & 7 & 5 & \multirow{3}{*}{0,545} \\
\hline & $1-2$ & 30 & 12 & \\
\hline & $3+$ & 50 & 30 & \\
\hline \multirow{3}{*}{ ABVDs } & Independente & 84 & 45 & \multirow{3}{*}{0,904} \\
\hline & Dependência parcial & 2 & 1 & \\
\hline & Dependência total & 1 & 1 & \\
\hline \multirow{3}{*}{ AIVDs } & Dependência total & 4 & 4 & \multirow{3}{*}{0,434} \\
\hline & Dependência parcial & 34 & 14 & \\
\hline & Independente & 49 & 29 & \\
\hline \multirow{2}{*}{ Envolvimento social } & Baixo & 58 & 30 & \multirow{2}{*}{0,442} \\
\hline & Alto & 29 & 17 & \\
\hline
\end{tabular}

Nota. ABVDs: atividades básicas de vida diária; AIVDs: atividades instrumentais de vida diária; p: significância estatística $(<0,05)$; * Teste qui-quadrado; \# Teste exato de Fisher

\section{Discussão}

Os resultados mostraram maior frequência de idosos homens vivendo em arranjos com a presença de um cônjuge ou companheira (só com ela ou com descendentes), enquanto as mulheres viviam a situação inversa, isto é, eram mais frequentes em arranjos sem a presença de um cônjuge. Idosos mais velhos (com idade igual ou superior a 75 anos) foram mais frequentes em arranjos com o cônjuge. Viver com um cônjuge também teve relação com menor número de doenças autorrelatadas.

Com a maior expectativa de vida entre as mulheres, espera-se que elas vivam mais do que seus companheiros. Por razões biológicas e comportamentais, os homens adoecem mais e com maior gravidade, o que aumenta a probabilidade de morte nesse grupo. Além disso, os homens têm maior chance de casamento e recasamento. Assim, mulheres idosas têm mais chances de viverem sozinhas ou só com seus descendentes (Campos et al., 2017; Neri, 2014).

Outros estudos identificaram que os homens tendem a viver com o cônjuge, enquanto as mulheres não, bem como a frequente chefia familiar do idoso (Camarano, Kanso, \& Mello, 2004; Gomes et al., 2013; Pedrazzi et al., 2010). Estudos demonstraram que viver com o cônjuge é um importante indicador de bem-estar na velhice (Campos et al., 2017; Chen \& Short, 2008; Del Duca et al., 2012; Russell \& Taylor, 2009). O apoio material e emocional oferecido nesse tipo de arranjo contribui para maior funcionalidade e longevidade. Essa vantagem é mais evidente entre os homens idosos, pois eles têm menores chances de se tornarem viúvos (Barreto, Giatti, \& Kalache, 2004; Camarano et al., 2004; Lebrão \& Laurenti, 2005).

Viver com um cônjuge parece ter efeito protetor sobre a saúde autorrelatada. Embora seja frequente a coexistência de uma ou mais doenças crônicas num mesmo indivíduo idoso, essa condição não está necessariamente associada à incapacidade ou fragilidade (Nunes et al., 2018). Um dado importante é de que três das quatro principais causas de internação hospitalar apontadas por Melo-Silva, Mambrini, Souza Junior, Andrade, e Lima-Costa (2018), a partir da base de dados do Estudo Longitudinal da Saúde dos Idosos Brasileiros (ELSI-Brasil), são objeto da atenção básica em saúde, sendo elas: acidente vascular cerebral, doença cardiovascular, diabetes e hipertensão. Essas doenças indicam uma importante demanda de cuidado.

É possível envelhecer de forma independente, autônoma e ativa mediante controle das doenças existentes, adotando medidas para a prevenção do declínio funcional e a manutenção da autonomia, especialmente quando favorecido pelo arranjo de moradia. Nesse contexto, as mulheres ainda são as maiores responsáveis pelo cuidado, e estudos indicam que essa tarefa está associada a piores índices de saúde física e bem-estar percebido (Giacomin et al., 2018; Neri, 2014).

A importância do cônjuge foi evidenciada pela associação desse arranjo com a idade, o sexo e o número de doenças crônicas. Idosos que vivem com o cônjuge estão mais protegidos contra morbidades e mortalidade (Lima-Costa et al., 2011; Manfredini \& Breschi, 2013). Quanto mais velho, maior a chance de viver sem o cônjuge (especialmente as mulheres). Nesse estudo, os mais velhos viviam mais com o cônjuge e isso provavelmente tem relação com a associação verificada com o menor número de doenças e com o sexo masculino. Os idosos que vivem sem o cônjuge constituem um grupo de risco, que requer atenção por parte dos investigadores, profissionais e gestores.

Maior envolvimento social foi observado nos arranjos mistos (com cônjuge e descendentes), o que pode significar que existe, nesse tipo de arranjo, um contexto mais favorável para o exercício da autonomia, o que libera espaço para o maior envolvimento social. As vantagens dos arranjos mistos têm sido apontadas por diversos estudos embasados no argumento de que as relações estabelecidas dentro do domicílio caracterizam-se pelo: suporte social material, físico ou emocional oferecido; maior probabilidade de manter-se ativo por meio da adoção de comportamentos de saúde considerados saudáveis; maior acesso 
aos serviços de saúde e menor risco de mortalidade associado à solidão e depressão (Cardoso, Sampaio, \& Vilela, 2017; Chen \& Short, 2008; Kooshiar et al., 2012; Russell \& Taylor, 2009). A compreensão dessa associação envolve o conhecimento sobre a qualidade das relações dentro do arranjo e sobre outros aspectos que podem justificar o envolvimento social nesses contextos, tais como renda, escolaridade, o papel de cuidador, carência de oportunidades e motivação para engajar-se em atividades sociais.

A participação social pode ser reduzida em função de problemas de saúde e incapacidade funcional, em decorrência das prioridades socioemocionais dos idosos ou devido a normas de comportamento etário, papéis de gênero e oportunidades sociais. No entanto estar socialmente envolvido intensifica o suporte social, aumenta o bem-estar, ajuda a manter o idoso autônomo no seu contexto sociocultural, promove um autoconceito mais positivo e aumenta o senso de controle sobre o ambiente e sobre a própria vida (Fontes et al., 2011; Neri, 2014). A participação social tem sido dificultada por características urbanas, como falta de acessibilidade e transporte público, consideradas importantes obstáculos para a vida cotidiana dos idosos, como identificado por Ferreira et al. (2018) a partir da base de dados ELSI- Brasil.

O grau de envolvimento social é um importante indicador de boas condições de saúde física e mental, além de ser preditor de satisfação com a vida na velhice (Neri, 2014; Pinto \& Neri, 2013). A participação em atividades sociais está condicionada também aos aspectos sociais e econômicos que envolvem os idosos, pois possibilitam ou dificultam o acesso às atividades sociais e de lazer. Na velhice, esses aspectos tendem a estar comprometidos, o que justifica, em parte, os baixos níveis de envolvimento social.

Nos arranjos em que os idosos vivem sozinhos, eles são os chefes de família e os maiores contribuintes com a renda familiar, pois esse tipo de arranjo exige certa independência financeira e funcional. Embora morar sozinho seja um tipo de arranjo que tem aumentado no Brasil (Freire \& Junior, 2017), essa condição pode tanto pressupor um envelhecimento saudável e autônomo como pode constituir risco para o idoso. Campos et al. (2017) verificaram que idosos vivendo sozinhos, mesmo que independentes, têm pior funcionalidade familiar.

Segundo dados do estudo Saúde, bem-estar e envelhecimento (SABE), a taxa de mortalidade é maior para os que vivem sozinhos quando comparada com os casados, em especial para os homens (Gomes et al., 2013). Campos et al. (2017) discutem que as relações de cuidado provavelmente estabelecidas no passado não garantem que idosos recebam a atenção devida pelos familiares. A melhor funcionalidade familiar encontrada naquele estudo foi entre os idosos homens casados com melhor condição social e de saúde.

A chefia familiar do idoso também foi mais frequente nos arranjos em que eles viviam com descendentes (com e sem o cônjuge). Quando se comparou os arranjos com descendentes e sem descendentes, observaram-se associações com os indicadores econômicos (arranjos com a presença de descendentes sem o cônjuge associaram-se com a maior contribuição financeira do idoso), o que significa que, mesmo vivendo com outras pessoas, os idosos são os responsáveis pelo sustento e o bem-estar da família. Viver com descendentes sem a presença do cônjuge pode representar sobrecarga adicional para o idoso (, 2014).

Em arranjos multigeracionais, ou seja, naqueles em que o idoso reside com filhos, netos e/ou bisnetos, há duas situações possíveis. Naquela em que os descendentes se encontram em dificuldades financeiras, o idoso ocupa o lugar de chefe de família, de proprietário da casa, sendo o maior contribuinte para a renda familiar. Outra situação é a de maior dependência do idoso devido a algum comprometimento da sua saúde física ou mental que afeta sua capacidade para realizar atividades diárias de maneira independente e segura, assim ele precisa de supervisão ou ajuda física de terceiros (Cardoso et al., 2017). Nota-se que, independente dos motivos que levam a constituição de um arranjo multigeracional, este tende a ser oneroso e colocar o idoso em situação de maior risco, inclusive pode se configurar numa violação se essa condição retirar algum direito da pessoa idosa.

Esse dado corrobora a ideia de que os descendentes migram para a moradia dos idosos em busca de apoio material, o que reflete uma situação social atual que tem implicações relevantes para o processo de envelhecimento. Numa fase em que o indivíduo requer atenção e cuidados da família, ele se mantém ou se torna o responsável pelo bem-estar dos outros. Nesse sentido, é possível que o idoso conviva com a falta de tempo e dinheiro para as suas necessidades e atividades, o que compromete sua adesão aos tratamentos de saúde e aos programas preventivos (Del Duca et al., 2012; Kasper et al., 2010; Lau \& Kirby, 2009).

Em arranjos multigeracionais, cujo idoso é o chefe da família e o provedor de recursos materiais, os agregados tornam-se dependentes do idoso, que, por sua vez, permanece sem tempo e dinheiro para cuidar da própria saúde (Camarano et al., 2004). Idosos responsáveis financeiramente pela família refletem arranjos formados provavelmente por sobrevivência e para maximização do apoio, o que coloca o idoso em maior vulnerabilidade social, uma vez que sua renda será dividida em seu prejuízo (Fontes et al., 2011). As condições financeiras, bem como o grau de preservação da saúde do idoso, são determinantes dos arranjos domiciliares.

Idosas que vivem sozinhas ou com membros da terceira e quarta gerações, e em pior condição de saúde, têm maior risco de viver em condições vulneráveis (Campos et al., 2017). Entre os fatores que determinam a saúde da população idosa em países em desenvolvimento, destacam-se as características do contexto social, que geram desigualdades na exposição aos riscos e vulnerabilidades e que mais interferem no bem-estar, na independência funcional e na qualidade de vida dos idosos (Andrade, Sena, Pinheiro, Meira, \& Lira, 2013; Geib, 2012; Giacomin et al., 2018; Lima-Costa, 2018).

Assim, o contexto social formado pela família pode ser importante para a etiologia social da saúde. Segundo Manfredini e Breschi (2013), em arranjos multigeracionais, a complexidade das relações, as desigualdades de gênero e uma possível 
concorrência por recursos entre os membros caracterizam esse contexto como de alta vulnerabilidade e alto risco para morbidade, declínio funcional e mortalidade de idosos.

Estar cercado por pessoas não garante os cuidados necessários na velhice, nem mesmo o apoio social e emocional que se espera. Muitas vezes, as relações familiares tendem a ser fontes de estresse, o que prejudica ainda mais a saúde e qualidade de vida (Cardoso et al., 2017). Lebrão e Laurenti (2005) verificaram que metade dos idosos relata que não recebem a ajuda que necessitam, o que revela a importância da qualidade das relações sociais e da disposição de recursos físicos, financeiros e sociais necessários para que o idoso seja cuidado no domicílio por seus membros.

\section{Considerações Finais}

Os homens tendem a viver acompanhados, seja pela esposa, seja pelos descendentes; os mais velhos vivem com o cônjuge; os que vivem sozinhos ou com descendentes tendem a ser os chefes de família e os maiores contribuintes com a renda familiar. Por fim, os idosos que vivem em arranjos mistos foram mais envolvidos socialmente quando comparados com os demais arranjos. Viver sozinho ou acompanhado, com ou sem descendentes, teve associações com indicadores sociodemográficos (chefia e contribuição financeira), entretanto viver com ou sem cônjuge foi associado com medidas de saúde, sexo e idade, o que pode indicar a importância desse membro familiar para o bem-estar do idoso. Entende-se, portanto, que os arranjos de moradia em que os idosos vivem, sozinhos ou em arranjos multigeracionais, podem refletir desvantagens sociais e contribuir para o declínio da saúde do idoso, o que caracteriza maior vulnerabilidade social.

Apesar das limitações inerentes a um trabalho de natureza descritiva, este estudo contribuiu para ampliar as discussões acerca dos diferentes tipos de arranjos de moradia como um indicador de vulnerabilidade social e suas associações com a saúde do idoso e os indicadores sociodemográficos. Entende-se que esses resultados têm relação com o bem-estar dos idosos, de seus familiares e dos cuidadores, bem como da sociedade, que deve se preparar para lidar com as demandas por cuidados na velhice.

Investigar os arranjos de moradia pela psicologia implica em reconhecer os aspectos objetivos e subjetivos avaliados pelos idosos envolvidos nas relações de troca no domicílio. Mais estudos populacionais e longitudinais, como com amostras maiores, são necessários para compreender alguns pontos ainda controversos sobre quais arranjos representam maior vulnerabilidade social para os idosos e para a identificação das condições que contribuem para o declínio funcional na velhice.

\section{Referências}

Andrade, L. M., Sena, E. L. D. S., Pinheiro, G. M. L., Meira, E. C., \& Lira, L. S. S. P. (2013). Políticas públicas para pessoas idosas no Brasil: Uma revisão integrativa. Ciência \& Saúde Coletiva, 18, 3543-3552. DOI: 10.1590/S141381232013001200011

Andrew, M. K., Mitnitski, A. B., \& Rockwood, K. (2008). Social vulnerability, frailty and mortality in elderly people. PLoS One, 3(5), e2232. DOI: 10.1371/journal.pone.0002232

Barreto, S. M., Giatti, L., \& Kalache, A. (2004). Gender inequalities in health among older Brazilian adults. Revista Panamericana de Salud Pública, 16, 110-117. Link

Camarano, A. A., Kanso, S., \& Mello, J. L. (2004). Como Vive o Idoso Brasileiro? In A. A. Camarano (Org.), Os novos idosos brasileiros muito além dos 60? (pp. 25-59). Rio de Janeiro: IPEA.

Campos, A. C. V., Rezende, G. P. D., Ferreira, E. F., Vargas, A. M. D., \& Gonçalves, L. H. T. (2017). Funcionalidade familiar de idosos brasileiros residentes em comunidade. Acta Paulista de Enfermagem, 30(4), 358-367. DOI: 10.1590/19820194201700053

Cardoso, L., Sampaio, T., \& Vilela, A. (2017). Cuidados fornecidos por familiares relacionados à convivência com o idoso. Revista Kairós: Gerontologia, 20(1), 353-367. DOI: 10.23925/2176-901X.2017v20i1p353-367

Carmo, M. E. D., \& Guizardi, F. L. (2018). O conceito de vulnerabilidade e seus sentidos para as políticas públicas de saúde e assistência social. Cadernos de Saúde Pública, 34, e00101417. DOI: 10.1590/0102-311X00101417

Chen, F., \& Short, S. E. (2008). Household context and subjective well-being among the oldest old in China. Journal of family issues, 29(10), 1379-1403. DOI: 10.1177/0192513X07313602 
Conselho Nacional de Saúde. (2012). Resolução do Conselho Nacional de Saúde 466/2012. Link

Del Duca, G. F., Martinez, A. D. D., \& Bastos, G. A. N. (2012). Perfil do idoso dependente de cuidado domiciliar em comunidades de baixo nível socioeconômico de Porto Alegre, Rio Grande do Sul. Ciência \& Saúde Coletiva, 17, 11591165. DOI: $10.1590 / \mathrm{S} 1413-81232012000500010$

Faleiros, A. H., Santos, C. A. D., Martins, C. R., Holanda, R. A. D., Souza, N. L. S. A. D., \& Araujo, C. L. D. O. (2015). Os desafios do cuidar: Revisão bibliográfia, sobrecargas e satisfações do cuidador de idosos. Janus, 12(22), 59-68. Link

Ferreira, F. R., César, C. C., Andrade, F. B. D., Souza, P. R. B. D., Junior, Lima-Costa, M. F., \& Proietti, F. A. (2018). Aspects of social participation and neighborhood perception: ELSI-Brazil. Revista de Saúde Pública, 52, 18s. DOI: 10.11606/ S1518-8787.2018052000647

Ferreira, M. (2017). Famílias e envelhecimento: um ensaio teórico com base na perspectiva histórica. Oikos: Família e Sociedade em Debate, 28(1), 143-161. Link

Fontes, A. P., Fortes-Burgos, A. C. G., Mello, D. M., Pereira, A. A., \& Neri, A. L. (2011). Arranjos domiciliares, expectativa de cuidado, suporte social percebido e satisfação com as relações sociais. In A. L. Neri \& M. E. Guariento (Orgs.), Fragilidade, saúde e bem-estar em idosos: Dados do estudo FIBRA Campinas (pp. 55-74). Campinas: Alínea.

Fontes, A. P., \& Neri, A. L. (2015). Resiliência e velhice: Revisão de literatura. Ciência \& Saúde Coletiva, 20 (5), 1475-1495. DOI: $10.1590 / 1413-81232015205.00502014$

Freire, R. D. M. H., \& Junior, N. C. (2017). Produção científica sobre habitação para idosos autônomos: Revisão integrativa da literatura. Revista Brasileira de Geriatria e Gerontologia, 20(5), 717-726. DOI: 10.1590/1981-22562017020.170065

Geib, L. T. C. (2012). Determinantes sociais da saúde do idoso. Ciência \& Saúde Coletiva, 17, 123-133. DOI: 10.1590/ S1413-81232012000100015

Giacomin, K. C., Duarte, Y. A. O., Camarano, A. A., Nunes, D. P., \& Fernandes, D. (2018). Cuidado e limitações funcionais em atividades cotidianas-ELSI-Brasil. Revista Saúde Pública, 52(Suppl 2). DOI: 10.11606/S1518-8787.2018052000650

Gomes, M. M. F., Turra, C. M., Fígoli, M. G. B., Duarte, Y. A., \& Lebrão, M. L. (2013). Associação entre mortalidade e estado marital: Uma análise para idosos residentes no Município de São Paulo, Brasil, Estudo SABE, 2000 e 2006. Cadernos de Saúde Pública, 29, 566-578. DOI: 10.1590/S0102-311X2013000300014

Kasper, J. D., Pezzin, L. E., \& Rice, J. B. (2010). Stability and changes in living arrangements: relationship to nursing home admission and timing of placement. Journals of Gerontology Series B: Psychological Sciences and Social Sciences, 65(6), 783-791. DOI: 10.1093/geronb/gbq023

Katz, S., Ford, A. B., Moskowitz, R. W., Jackson, B. A., \& Jaffe, M. W. (1963). Studies of illness in the aged: The index of ADL: A standardized measure of biological and psychosocial function. Jama, 185(12), 914-919. Link

Kooshiar, H., Yahaya, N., Hamid, T. A., Samah, A. A., \& Jou, V. S. (2012). Living arrangement and life satisfaction in older Malaysians: The mediating role of social support function. PloS one, 7(8), e43125. DOI: 10.1371/journal.pone.0043125

Lau, D. T., \& Kirby, J. B. (2009). The relationship between living arrangement and preventive care use among communitydwelling elderly persons. American Journal of Public Health, 99(7), 1315-1321. DOI: 10.2105/AJPH.2008.151142

Lawton, M. P., \& Brody, E. M. (1969). Assessment of older people: self-maintaining and instrumental activities of daily living. The gerontologist, 9(3_Part_1), 179-186. Link

Lebrão, M. L., \& Laurenti, R. (2005). Saúde, bem-estar e envelhecimento: O estudo SABE no Município de São Paulo. Revista brasileira de epidemiologia, 8, 127-141. Link 
Lima-Costa, M. F. (2018). Envelhecimento e saúde coletiva: Estudo Longitudinal da Saúde dos Idosos Brasileiros (ELSIBrasil). Revista de Saúde Pública, 52(Supl 2), 2s. DOI: 10.11606/s1518-8787.201805200supl2ap

Lima-Costa, M. F., Matos, D. L., Camargos, V. P., \& Macinko, J. (2011). Tendências em dez anos das condições de saúde de idosos brasileiros: Evidências da Pesquisa Nacional por Amostra de Domicílios (1998, 2003, 2008). Ciência \& saúde coletiva, 16, 3689-3696. Link

Manfredini, M., \& Breschi, M. (2013). Living arrangements and the elderly: An analysis of old-age mortality by household structure in Casalguidi, 1819-1859. Demography, 50(5), 1593-1613. DOI: 10.1007/s13524-013-0218-0

Melo-Silva, A. M. D., Mambrini, J. V. D. M., Souza, P. R. B. D., Junior, Andrade, F. B. D., \& Lima-Costa, M. F. (2018). Hospitalizations among older adults: Results from ELSI-Brazil. Revista de Saúde Pública, 52, 3s. DOI: 10.11606/ S1518-8787.2018052000639

Neri, A. L. (2014). Palavras-chave em Gerontologia. Campinas, SP: Alínea.

Neri, A. L., Borim, F. S. A., Fontes, A. P., Rabello, D. F., Cachioni, M., Batistoni, S. S. T., ... \& Lima-Costa, M. F. (2018). Fatores associados à qualidade de vida percebida em adultos mais velhos: ELSI-Brasil. Revista Saúde Pública, 52 (Suppl 2),16s. DOI: 10.11606/s1518-8787.2018052000613

Nunes, B. P., Batista, S. R. R., Andrade, F. B. D., Souza, P. R. B. D., Junior, Lima-Costa, M. F., \& Facchini, L. A. (2018). Multimorbidity: The brazilian longitudinal study of aging (ELSI-Brazil). Revista de Saúde Pública, 52, 10s. DOI: 10.11606/S1518-8787.2018052000637

Pavarini, S., Barha, E., Mendiondo, M., Filizola, C., Petrilli, J., Filho, \& Santos, A. (2009). Família e vulnerabilidade social: Um estudo com octogenários. Revista Latino-Americana De Enfermagem, 17(3), 374-379. DOI: 10.1590/S010411692009000300015

Pedrazzi, E. C., Motta, T. D., Vendrúscolo, T. R. P., Fabrício-Wehbe, S. C., Cruz, I. R., \& Rodrigues, R. A. P. (2010). Arranjo domiciliar dos idosos mais velhos. Revista latino-americana de enfermagem, 18(1), 18-25. DOI: 10.1590/ S0104-11692010000100004

Perseguino, M., Horta, A., \& Ribeiro, C. (2017). A família frente a realidade do idoso de morar sozinho. Revista Brasileira de Enfermagem, 70 (2), 251-257. DOI: 10.1590/0034-7167-2016-0398

Pinto, J. M., \& Neri, A. L. (2013). Factors associated with low life life satisfaction in community-dwelling elderly: FIBRA Study. Cadernos de Saúde Pública, 29, 2447-2458. Link

Russell, D., \& Taylor, J. (2009). Living alone and depressive symptoms: The influence of gender, physical disability, and social support among Hispanic and non-Hispanic older adults. Journals of Gerontology Series B: Psychological Sciences and Social Sciences, 64(1), 95-104. DOI: 10.1093/geronb/gbn002

Salmazo-Silva, H., Lima-Silva, T. B., de Barros, T. C., de Oliveira, E. M., Ordonez, T. N., Carvalho, G., \& de Almeida, E. B. (2012). Vulnerabilidade na velhice: Definição e intervenções no campo da Gerontologia. Revista Kairós: Gerontologia, 15, 97-116. Link

Wang, H., Chen, K., Pan, Y., Jing, F., \& Liu, H. (2013). Associations and impact factors between living arrangements and functional disability among older Chinese adults. PLoS One, 8(1), e53879. DOI: 10.1371/journal.pone.0053879 


\section{Como citar:}

Dóris, D. F., Rocha, N. M. F. D., \& Pinto, J. M. (2020). Arranjos de Moradia de Idosos: Associação com Indicadores Sociodemográficos e de Saúde. Revista Subjetividades, 20(Esp 1. Relações Intergrupais: Preconceito e Exclusão Social), e8873. http://doi.org/10.5020/23590777.rs.v20iEsp1.e8873

\section{Endereço para correspondência}

Dóris Firmino Rabelo

E-mail: drisrabelo@yahoo.com.br

Nara Maria Forte Diogo Rocha

E-mail: narafdiogo@gmail.com

Juliana Martins Pinto

E-mail: ju_fisio33@yahoo.com.br 